\section{Intensity-modulated radiotherapy for relapsed malignant pleural mesothelioma}

\author{
Corrado Spatola*,1, Carmelo Militello', Alessandra Tocco', Vincenzo Salamone1, \\ Luigi Raffaele', Marcello Migliore ${ }^{2}$, Antonio Pagana', Roberto Milazzotto', \\ Ilenia Chillura', Stefano Pergolizzi ${ }^{3} \&$ Giuseppe Privitera'
}

The use of novel radiotherapy techniques is widely increasing, allowing clinicians to treat diseases that were previously difficult to treat with radiation therapy. Malignant pleural mesothelioma is a clear example of this clinical challenge. We describe our first experience with intensity-modulated radiotherapy technique which was used to treat a 73-year-old patient with multiple relapsing malignant pleural mesothelioma. Intensity-modulated radiation therapy has allowed to respect the QUANTEC (quantitative analyses of normal tissue effects in the clinic) dose constraints, patient has experienced a 14 months progression-free time, without relevant subacute or late lung toxicity.

First draft submitted: 14 July 2016; Accepted for publication: 7 September 2016; Published online: 21 September 2016

Malignant pleural mesothelioma (MPM) is a serious challenge for the radiation oncologists, because of its natural history of high tendency to local relapse, most often confined to the ipsilateral pleura. Surgery is considered the treatment of choice, by means of an extrapleural pneumonectomy (EPP) or pleurectomy/decortication, but the rate of local control is very low in local advanced disease and, consequently, a local radical therapy is often needed in order to prolong the time to progression [1,2]. In this setting, 3D conventional external beam radiotherapy encounters great difficulties, due to the large radiation fields required, resulting in increased risk of lung toxicity [3,4].

We report on our first experience with a complex intensity-modulated radiation therapy (IMRT) technique, used in a patient with a history of multiple relapsed locally advanced MPM.

\section{Patient history}

A 73-year-old man was referred to our department in September 2015 with a diagnosis of relapsed MPM of the right pleura. He had previously received an EPP on October 2013 (histologic subtype: epithelioid) with hyperthermic intrapleural chemotherapy with cisplatin, followed by an adjuvant chemotherapy with carboplatin-pemetrexed for six cycles. No adjuvant radiotherapy was proposed. After a disease-free survival of 10 months, a local relapse infiltrating circumferentially the right lung was detected on a computed tomography-fludeoxyglucose-PET (CT-18FDG-PET). After a chemotherapy rechallenge with the same protocol for two cycles (with no disease response), a local irradiation was proposed on June 2015. The patient was assessed according to modified RECIST criteria before and after radiotherapy. We defined a target volume (clinical target volume [CTV]

'UOC Radiodiagnostica e Radioterapia, AOU Policlinico-VE di Catania, 95125, Italy

${ }^{2}$ UOC Chirurgia Toracica, AOU Policlinico-VE Catania

${ }^{3}$ UOC Radioterapia, AOU Policlinico di Messina, 98124, Italy

*Author for correspondence: cor_spatola@hotmail.com

\section{KEYWORDS}

- IMRT • malignant pleural mesothelioma $\bullet$ radiation therapy 
1) as a ring encompassing the whole right lung composed of the pleura and the chest wall of the entire hemithorax, with a dose of $5040 \mathrm{cGy}$ in 28 fractions. A second volume (CTV2) was identified, composed by a gross visible disease located at the lung apex, with a dose of $1080 \mathrm{cGy}$ in six fractions. PTV1 and PTV2 were created by adding $1 \mathrm{~cm}$ circumferential margins to CTV1 and CTV2, to account for respiratory movements. A treatment plan comparison was made between a conventional tridimensional conformal radiotherapy (3D-CRT) technique, through two AP-PA (antero-posterior portals) opposed fields and an IMRT, through eight static 'step and shoot' fields (at $20^{\circ}, 150^{\circ}, 180^{\circ}$, $220^{\circ}, 270^{\circ}, 300^{\circ}, 340^{\circ}, 350^{\circ}$ angles, with a total of 131 segments, see Figure 1 ). The IMRT plan was preferred based on the resulting doses to the organs at risk (OARs), according to the QUANTEC (quantitative analyses of normal tissue effects in the clinic) constraints. The treatment plan was developed by treatment planning system Elekta XiO (Elekta AB, Stockholm,
Sweden). The Dosimetry Check software (Math Resolutions, LLC5975 Gales Lane, Columbia) provides patient-specific pretreatment dose quality assurance of intensity modulated treatment plan. The $\gamma$ index passing rate $(3 \%, 3 \mathrm{~mm})$ was 96\% of measured points. The doses at OARs were calculated by treatment planning system and are the following:

- Spinal cord: mean dose 1841 cGy, $\mathrm{D}_{\max }$ 4200 cGy;

- Heart: V25 4.24\%, V30 2.2\%;

- Liver: mean dose 951 cGy;

- Left lung: V20 0.14\%;

- Right lung: V20 38\%;

- Combined lung: V20 29\%, mean dose 2210 cGy.

\section{- Esophagus: V35 24.63\%}

The patient was able to complete treatment with medical support and without the need

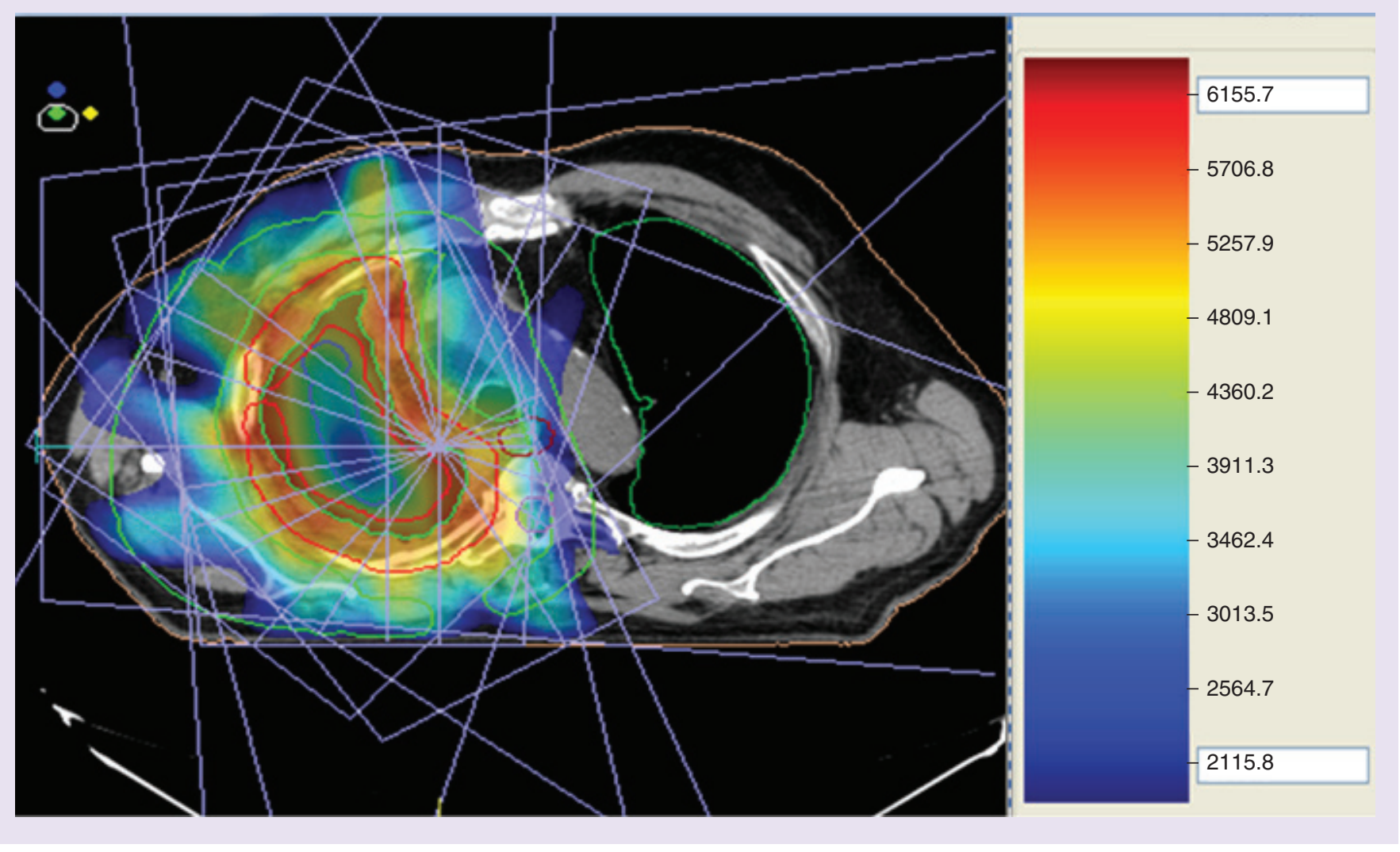

Figure 1. Treatment planning dose distribution with static step and shoot intensity-modulated radiation therapy technique. Red contour indicate the clinical target volume 1, green contour (outer to red one) indicate a ghost volume for treatment planning procedures, lung volumes are indicated with inner green contours. 
for treatment breaks. No grade III toxicity was detected, but only a grade II esophagitis. At a follow-up time of 14 months, he was free from progression and without any relevant grade II or more subacute or late lung toxicity.

\section{Discussion}

The role and the timing of radiotherapy in the management of MPM is still controversial, because, despite it being widely used both in postoperative and in radical setting, the risk of lung and heart toxicity pose a serious restriction or delay in its clinical application $[2,3]$. There are also a lack of randomized trials assessing the use of radiotherapy after EPP. Literature reports on several small series where radiotherapy is a part of trimodality approach in adjuvant setting and as a palliative treatment in recurrent or advanced diverse [5-7].

In the few last years, the technological advancements in delivery of radiation therapy has permitted to use high radiation doses to target volumes in the thorax, while maintaining the dose to the surrounding normal tissues (liver, heart, cord and lung above all) under the tolerance limits. IMRT technique can reduce dose to OARs, with a small and clinically negligible increase in dose absorption to the contralateral lung, compared with conventional 3D-CRT technique. To date, the role of IMRT in the treatment of MPM is not supported by clear evidence, and its use is limited inside an experimental series [4-8].

The patient on whom, we report is an elderly man with a history of relapsed MPM. Despite the age and the previous thoracic surgery, his pulmonary function tests (FEV1 and DLCO) were within normal limits at the time of enrollment. The patient was admitted at the radiotherapy department 12 months after surgery, with a local relapse not responding to chemotherapy. IMRT technique was preferred to conventional 3D-CRT because of a more favorable dose-volume histograms developed during the planning process. In particular doses to the spinal cord, to the contralateral lung and to the esophagus were within the QUANTEC dose constraints, and this explains the good acute and subacute tolerance to radiotherapy [9]. The major challenge was the dose to the affected lung, which showed a V20 of 38\%, above the QUANTEC constraint, but, despite the high risk, no relevant grade II-IV toxicity have been detected at a follow-up time of 10 months. The patient is still alive after 32 months from diagnosis, and, at the time of this report, is free from local and distant progression.

The reported experiences on the use of thoracic IMRT for MPM show unclear data on the outcome. The majority of trials regarding the use of radiotherapy as a component of radical treatment, both in a trimodality approach and as an adjuvant therapy after EPP, showed with IMRT a reduced dose to OARs such liver, hearth, kidney and affected lung, but an increased dose to contralateral lung and kidney [4-8]. Moreover, some authors reported serious adverse events, like the Boston experience, where six of 13 patients treated with IMRT after EPP (dose 5400 cGy, with a V20 >20\%) died from radiation pneumonitis [10]. Also, a large prospective randomized Phase II trial (SAKK 17/04) on multimodality therapy showed that the addition of hemithoracic radiation to chemotherapy and surgery failed to improve local control and overall survival in MPM [11]. For these reasons, currently, the use of IMRT must be limited to clinical trials, especially in adjuvant setting after EPP, where more prospective studies are needed to determine the safe dose levels to be respected, while its role in palliative setting, included relapsed disease and pain control, is well established.

\section{Conclusion}

IMRT of locally advanced or relapsing MPM is a feasible treatment option, with an acceptable toxicity profile. In our experience, we have used a complex static step and shoot technique, which allowed us to maintain the dose to the OARs under the constraints of QUANTEC. The conventional 3D-CRT technique is usually unable to safely deliver a tumoricidal dose of about 5000 cGy. This is only a case report and more studies are needed to validate an extended use of IMRT in a challenging disease such as MPM.

\section{Financial \& competing interests disclosure}

The authors have no relevant affiliations or financial involvement with any organization or entity with a financial interest in or financial conflict with the subject matter or materials discussed in the manuscript. This includes employment, consultancies, honoraria, stock ownership or options, expert testimony, grants or patents received or pending, or royalties.

No writing assistance was utilized in the production of this manuscript. 
Informed consent disclosure

The authors state that they have obtained verbal and written informed consent from the patient/patients for the inclusion of their medical and treatment history within this case report.
Open access

This work is licensed under the Creative Commons Attribution-NonCommercial 4.0 Unported License. To view a copy of this license, visit http://creativecommons.org/ licenses/by-nc-nd/4.0/

\section{EXECUTIVE SUMMARY}

- Malignant pleural mesothelioma (MPM) is a serious challenge for the radiation oncologist, because of its natural history of high tendency to local relapse. Surgery is considered the treatment of choice, but a local radical therapy is often needed in order to prolong the time to progression.

- This manuscript report authors first experience with a complex intensity-modulated radiation therapy (IMRT) technique, used in a patient with a history of multiple relapsed locally advanced MPM, whereas 3D conventional external beam radiotherapy does not allow to achieve the same therapeutic goals.

- A 73-year-old man was referred to the Radiotherapy Department, after he underwent to extrapleural pneumonectomy (EPP), with hyperthermic intrapleural chemotherapy with cisplatin, for a right epithelioid MPM. Surgery was followed by adjuvant chemotherapy with carboplatin-pemetrexed for six cycles.

- At the time of recruitment, the patient had a local relapse infiltrating circumferentially the right lung. A local radiotherapy was proposed.

- A treatment plan comparison was made between a conventional 3D-conformal radiotherapy technique and an IMRT.

- The IMRT plan was preferred based on the resulting doses to the organs at risk (OARs), according to the QUANTEC constraints. IMRT was done through eight static 'step and shoot' fields.

- Clinical target volume 1 was defined as a ring encompassing the whole right lung composed of the pleura and the chest wall of the entire hemithorax, with a dose of 5040 cGy in 28 fractions. A second volume (clinical target volume 2) was identified, composed by a gross visible disease located at the lung apex, with a dose of 1080 cGy in six fractions.

- The doses at OARs were calculated by treatment planning system and are the following: spinal cord: mean dose 1841 cGy, D $\max 4200$ cGy; heart: V25 4.24\%, V30 2.2\%; liver: mean dose 951 cGy; left lung: V20 0.14\%; right lung: V20 38\%; combined lung: V20 29\%, mean dose 2210 cGy; esophagus: V35 24.63\%.

- The patient was able to complete treatment. No grade III toxicity was detected, but only a grade II esophagitis. At a follow-up time of 14 months, he was free from progression and without any relevant grade II or more subacute or late lung toxicity.

- The reported experiences on the use of thoracic IMRT for MPM show unclear data on the outcome. The majority of trials regarding the use of radiotherapy as a component of radical treatment, both in a trimodality approach and as an adjuvant therapy after EPP, showed with IMRT a reduced dose to OARs such liver, hearth, kidney and affected lung, but an increased dose to contralateral lung and kidney.

- Currently, the use of IMRT must be limited to clinical trials, especially in adjuvant setting after EPP, where more prospective studies are needed to determine the safe dose levels to be respected, while its role in palliative setting, included relapsed disease and pain control, is well established.

- Manuscript concluded that IMRT of locally advanced or relapsing MPM is a feasible treatment option, with an acceptable toxicity profile.

\section{References}

Papers of special note have been highlighted as:

- of interest; $\bullet$ of considerable interest

1 Migliore M, Calvo D, Criscione A et al. Cytoreductivesurgery and hyperthermic intrapleural chemotherapy for malignant pleuraldiseases: preliminary experience.

Future Oncol. 11, 47-52 (2015).

- Regarding the general management of malignant pleural mesothelioma (MPM), especially about the treatment strategy and the timing of interventions.
2 Price A. What is the role of radiotherapy in malignant pleural mesothelioma? Oncologist 16, 359-365 (2011).

3 Yajnik S, Rosenzweig KE, Mychalczak B et al. Hemithoracic radiation after extrapleural pneumonectomy for malignant pleural 
mesothelioma. Int. J. Radiat. Oncol. Biol. Phys. 56, 1319-1326 (2003).

4 Tonoli S, Vitali P, Scotti V et al. Adjuvant radiotherapy after extrapleural pneumonectomy for mesothelioma. Prospective analysis of a multi-institutional series. Radiother. Oncol. 101, 311-315 (2011).

- Regarding the general management of MPM, especially about the treatment strategy and the timing of interventions.

5 Rosenzweig KE, Zauderer MG, Laser B et al. Pleural intensity-modulated radiotherapy for malignant pleural mesothelioma. Int. J. Radiat. Oncol. Biol. Phys. 83, 1278-1283 (2012).

-. Regarding the feasibility and technical specifications of intensity-modulated radiation therapy (IMRT) for MPM.

6 Ahamad A, Stevens CW, Smythe WR et al. Intensity-modulated radiation therapy: a novel approach to the management of malignant pleural mesothelioma. Int. J. Radiat. Oncol. Biol. Phys. 55, 768-775 (2003).

-. Regarding the feasibility and technical specifications of IMRT for MPM.

7 Sterzing F, Sroka-Perez G, Schubert K et al. Evaluating target coverage and normal tissue sparing in the adjuvant radiotherapy of malignant pleural mesothelioma: helical tomotherapy compared with step-and-shoot IMRT. Radiother. Oncol. 86, 251-257 (2008).

-. Regarding the feasibility and technical specifications of IMRT for MPM.

8 Rimner A, Rosenzweig KE. Novel radiation therapy approaches in malignant pleural mesothelioma. Ann. Cardiothorac. Surg. 1(4), 457-461 (2012).

-. Regarding the feasibility and technical specifications of IMRT for MPM.

9 Marks B, Bentzen SM et al. Radiation dose-volume effects in the lung. Int. J.
Radiat. Oncol. Biol. Phys. 76(Suppl. 3), S70-S76 (2010).

10 Allen AM, Czerminska M, Janne PA et al. Fatal pneumonitis associated with intensitymodulated radiation therapy for mesothelioma. Int. J. Radiat. Oncol. Biol. Phys. 65, 640-645 (2006).

-. Regarding the feasibility and technical specifications of IMRT for MPM.

11 Stahel R, Riesterer O, Alexandros X et al. Neoadjuvant chemotherapy and extrapleural pneumonectomy of malignant pleural mesothelioma with or without hemithoracic radiotherapy: final results of the randomized multicenter Phase II trial SAKK 17/04.

Presented at: ESMO 2014 Congress. Madrid, Spain, 26-30 September 2014 (Abstract LBA37_PR).

- Regarding the general management of MPM, especially about the treatment strategy and the timing of interventions. 